\title{
Rapid alkaline methylene blue supravital staining for assessment of anterior segment infections
}

\author{
This article was published in the following Dove Press journal: \\ Clinical Ophthalmology \\ II October 2016 \\ Number of times this article has been viewed
}

\author{
Katsuji Kiuchi \\ Kiuchi Eye Clinic, Joto-ku, \\ Osaka, Japan
}

Correspondence: Katsuji Kiuchi

Kiuchi Eye Clinic, 6-8-16

Higashinakahama, Joto-ku,

Osaka 536-0023, Japan

Tel +8I 669623736

Fax +8I 669684984

Email kkiuchil@yahoo.co.jp
Purpose: To present the Löffler's alkaline methylene blue technique of staining eye discharges in eyes with anterior segment infections.

Method: The Löffler's alkaline methylene blue staining method is a simple staining technique that can be used to differentiate bacterial, viral, and fungal infections. It is a cationic dye that stains cells blue because the positively charged dye is attracted to negatively charged particles such as polyphosphates, DNAs, and RNAs. Specimens collected from patients by swabbing are smeared onto microscope slides and the methylene blue solution is dropped on the slide. The slide is covered with a glass cover slip and examined under a microscope. The entire time from the collection to the viewing is about 30 seconds.

Results: Histopathological images of the conjunctival epithelial cells and neutrophils in eye discharges were dyed blue and the nuclei were stained more intensely blue. Bacterial infections consisted mainly of neutrophils, and viral infections consisted mainly of lymphocytes.

Conclusions: Löffler's alkaline methylene blue staining can be done in about 30 seconds for diagnosis. Even though this is a one color stain, it is possible to infer the cause of the infection by detection of the absence of bacteria and/or fungi in context of the differential distribution of neutrophils and lymphocytes.

Keywords: alkaline methylene blue, supravital staining, discharge, anterior segment infections

\section{Introduction}

Conjunctivitis is one of the most common diseases encountered by ophthalmologists in their daily practice. Most bacterial conjunctivitis is self-limiting although topical antibiotics are recommended because they can shorten the duration of the disease and prevent the spread of the infection. Although identifying the microorganism causing the conjunctivitis should be the first step in the treatment protocol, most conventional staining procedures require a long time. Thus, broad-spectrum antibiotics are generally used empirically as the first-line therapy for bacterial conjunctivitis. Topical antibiotics are recommended to deliver high levels of the drug directly to the site of infection. But the optimal antibiotic cannot be determined without more extensive culturing, isolation, identification, and sensitivity procedures. Thus, a rapid technique for a tentative identification of the causative microorganism is needed.

Conjunctivitis can be caused by bacteria, viruses, or fungi. ${ }^{1}$ Clinically, conjunctivitis is manifested by conjunctival hyperemia, excessive tearing, eye discharge, conjunctival follicles, and pseudomembrane formation. ${ }^{2}$ It is, however, difficult to determine the causative microorganism by the signs and symptoms alone.

It is generally known that conjunctivitis caused by bacteria has a predominant infiltration by neutrophils, whereas conjunctivitis caused by viruses has a predominance of lymphocytes. ${ }^{3,4}$ Although staphylococci, eg, Haemophilus influenzae, are considered 
to be the main cause of bacterial conjunctivitis, ${ }^{3,5}$ it is necessary to use Gram or Giemsa staining as a generally accepted method for the final diagnosis. However, the procedures for these staining methods require several steps and several days.

Thus, the Diff-Quik stain has been recommended as a simpler and quicker method of staining specimens of eyes with conjunctivitis. Diff-Quik method is a commercial Romanowsky stain variant which is based on a modification of the Wright-Giemsa stain. Even though Diff-Quik stain is simpler, it still requires many steps including smearing of the specimen, fixing, staining, clearing, and it is difficult to perform during a busy ophthalmic examination schedule.

The purpose of this report is to present the procedures used in the Löffler's alkaline methylene blue staining for eyes with anterior segment infections. This method is currently used in our clinic as an auxiliary diagnostic method for eyes with conjunctivitis to determine whether it is bacterial or viral conjunctivitis by judging the different infiltration by neutrophils and lymphocytes.

\section{Materials and methods}

The Löffler's alkaline methylene blue (Muto Pure Chemicals Co, Ltd Bunkyo-ku, Tokyo, Japan) solution is made by a tenfold dilution of the original solution with distilled water or saline, and it is filtered by Millex AP Filter, pore size $2.0 \mu \mathrm{m}$ (Millipore: SLAP 02550; Merck KGaA, Darmstadt, Germany) before use every day. The eye discharge is collected with a sterile cotton swab and spread thinly on a microscope slide. One drop of the Löffler's alkaline methylene blue solution is dripped onto the specimen, cover slipped, and examined by a conventional optical microscope immediately. The whole procedure takes about 30 seconds.
As control, specimens were collected in the same way but stained with the Diff-Quik method. Eye discharge was collected from 17 patients whose mean age was $76.8 \pm 22.5$ years ( \pm standard deviation) with a range from 6 months to 94 years (Table 1). All patients signed written informed consent for participating in this study. One patient had chronic conjunctivitis, 3 had acute conjunctivitis, 3 had dacryocystitis, and 10 had blepharitis. The time required to perform the Löffler's alkaline methylene blue method was determined and compared to the time required for the Diff-Quik method. Ethics committee approval was obtained from the institutional review board of Kiuchi Eye Clinic. This study was performed in accordance with the ethical tenets outlined in the Declaration of Helsinki.

\section{Results}

Photomicrographs of the bacteria in the eye discharge of a patient with chronic conjunctivitis are shown in Figure 1A and B. The discharge shown in Figure 1A was stained by the Löffler's alkaline methylene blue method and the discharge shown in Figure 1B was stained by the Diff-Quik method. The bacteria are diplococci and are stained blue. The photomicrographs of neutrophils and lymphocytes in the eye discharge from a patient with blepharitis are shown in Figure 2A and B. The specimen shown in Figure 2A was stained by the Löffler's alkaline methylene blue method and that shown in Figure 2B was stained by the Diff-Quik method. Some of the neutrophils have segmented figures. Although the Löffler's alkaline methylene blue staining is a one color, the quality of staining is as good as the Diff-Quik method.

Photomicrographs of lymphocytes in the eye discharge from a patient with acute conjunctivitis are shown in Figure 3A and B. In Figure 3A, the eye discharge was stained by the Löffler's alkaline methylene blue method and that

Table I Summary of patient diagnosis and characteristics

\begin{tabular}{lllll}
\hline Case & Age (years) & Sex & Diagnosis & Microscopic finding \\
\hline 1 & 0.5 & Male & Acute conjunctivitis & Mainly lymphocytes \\
2 & 51 & Male & Acute conjunctivitis & Mainly lymphocytes \\
3 & 64 & Female & Acute conjunctivitis & Mainly lymphocytes \\
4 & 81 & Female & Chronic conjunctivitis & Mainly lymphocytes \\
5 & 76 & Female & Chronic dacryocystitis & Mainly neutrophils \\
6 & 79 & Female & Chronic dacryocystitis & Mainly neutrophils \\
7 & 82 & Female & Chronic dacryocystitis & Mainly neutrophils \\
8 & 78 & Female & Blepharitis & Mainly neutrophils \\
9 & 80 & Female & Blepharitis & Mainly neutrophils \\
10 & 83 & Male & Blepharitis & Mainly neutrophils \\
11 & 88 & Female & Blepharitis & Mainly neutrophils \\
12 & 89 & Female & Blepharitis & Mainly neutrophils \\
13 & 89 & Female & Blepharitis & Mainly neutrophils \\
14 & 89 & Female & Blepharitis & Mainly neutrophils \\
15 & 91 & Female & Blepharitis & Mainly neutrophils \\
16 & 91 & Male & Blepharitis & Mainly neutrophils \\
17 & 94 & Female & & Mainly neutrophils \\
\hline
\end{tabular}



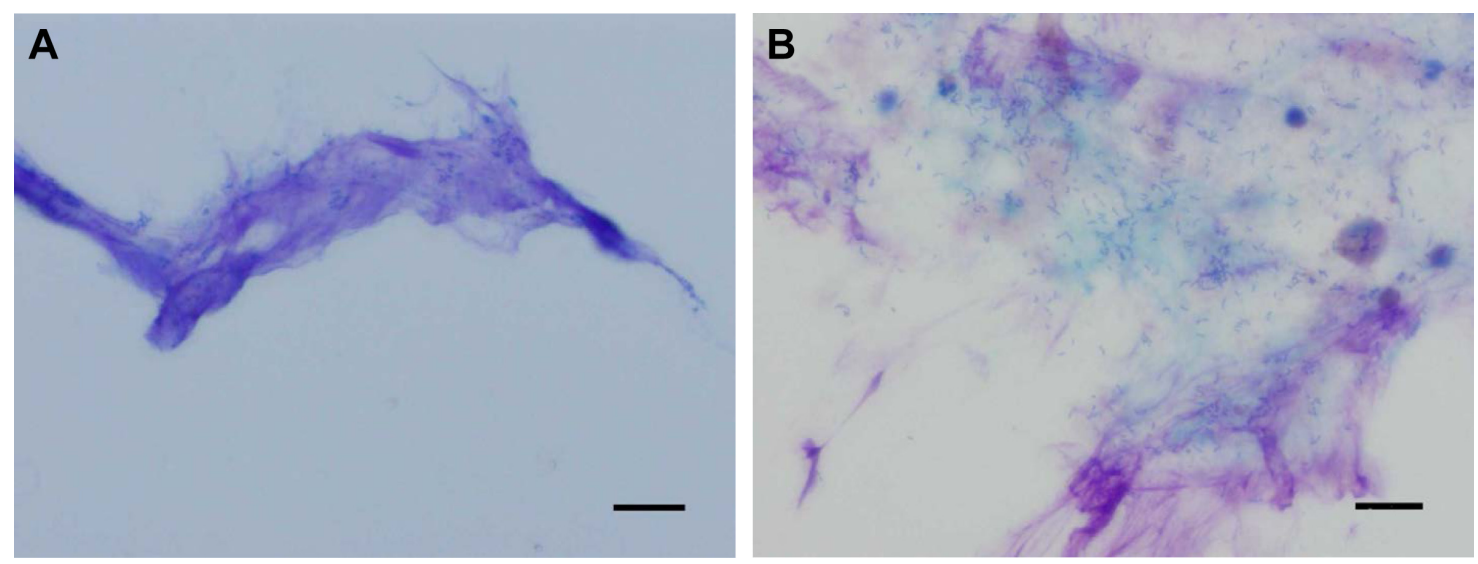

Figure I Photomicrograph of a histopathological specimen stained with the Löffler's alkaline methylene blue method (A) and Diff-Quik method (B).

Notes: The specimen was collected from an eye with chronic conjunctivitis of an 81 -year-old female. The bacteria are diplococci and are stained blue. Bar $=200 \mu \mathrm{m}$. The conventional optical microscope magnification is $\times 400$.
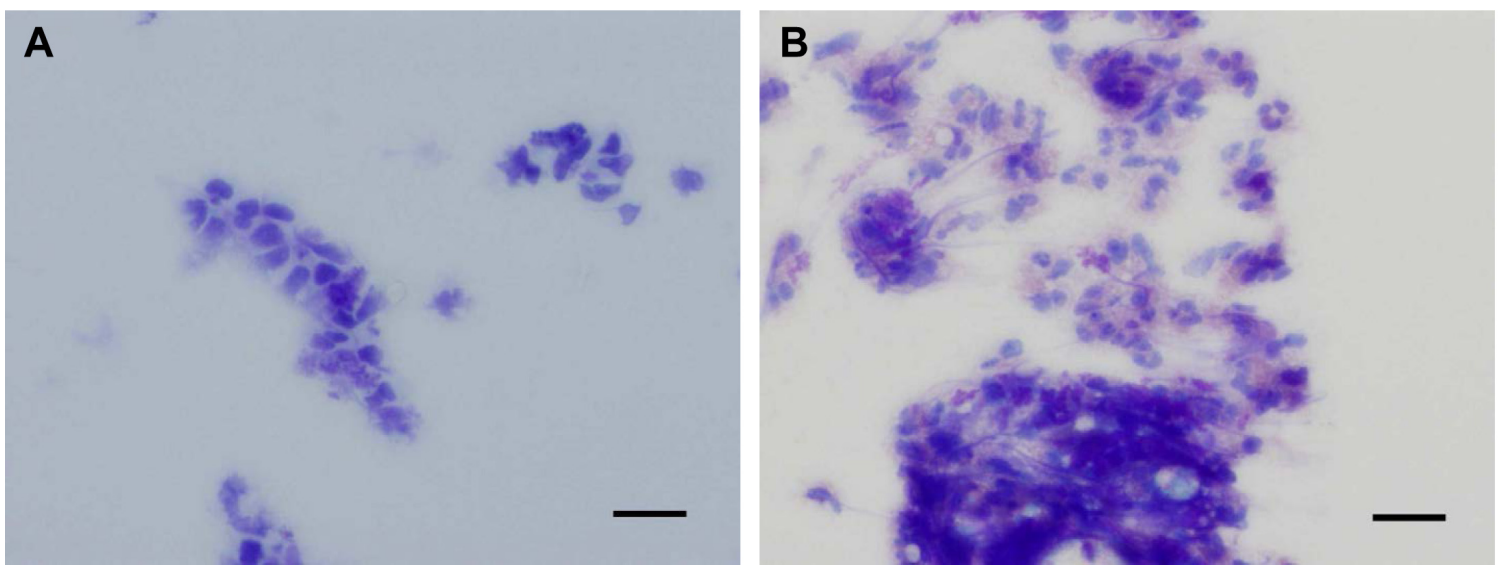

Figure 2 Photomicrographs of a histopathological specimen stained with the Löffler's alkaline methylene blue method (A) and Diff-Quik method (B).

Notes: The specimen was collected from an eye with blepharitis of an 88-year-old female. Many neutrophils are present in the eye discharge. Bar $=200 \mu \mathrm{m}$. The conventional optical microscope magnification is $\times 400$.
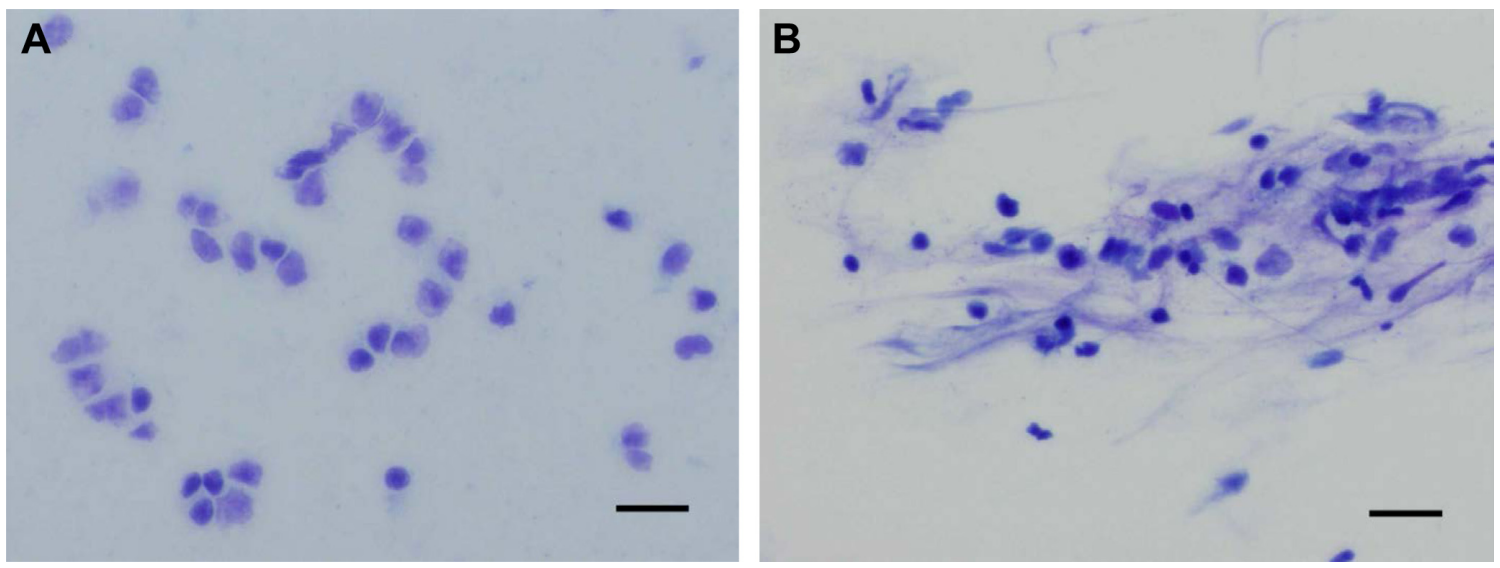

Figure 3 Photomicrographs of a histopathological specimen stained with the Löffler's alkaline methylene blue method (A) and Diff-Quik method (B).

Notes: The specimen was collected from an eye with acute conjunctivitis of a 6-month-old male. Many lymphocytes are present in the eye discharge. Bar $=200 \mu \mathrm{m}$. The conventional optical microscope magnification is $\times 400$. 


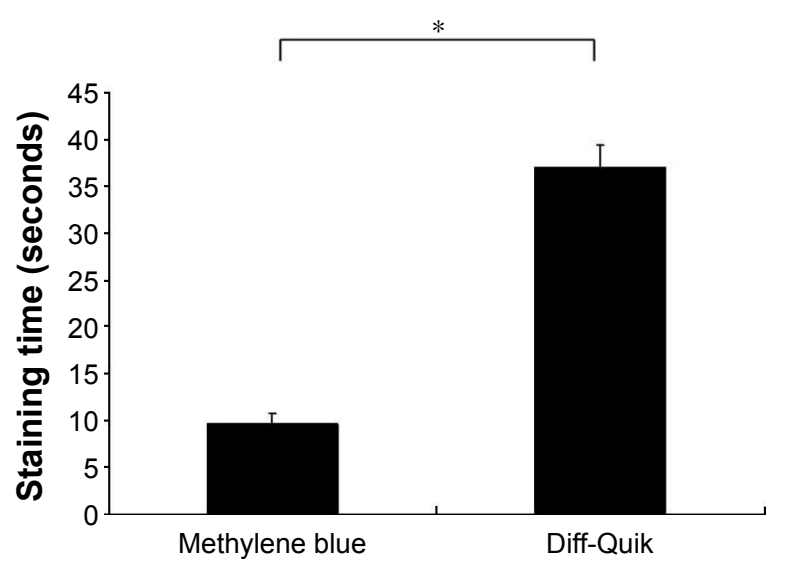

Figure 4 Comparison of staining time of Löfler's alkaline methylene blue method and Diff-Quik method.

Notes: Staining time of methylene blue method is $9.7 \pm 4.59$ seconds, while the staining time of Diff-Quik method is $37.1 \pm 9.36$ seconds ( \pm standard deviation; $* P<0.01$ paired $t$-test).

shown in Figure 3B was stained by the Diff-Quik method. Many lymphocytes are seen, and the quality of staining with the Löffler's alkaline methylene blue method is as good as with the Diff-Quik method.

The staining time of the Löffler's alkaline methylene blue method was $9.7 \pm 4.59$ seconds and that for the Diff-Quik method was $37.1 \pm 9.36$ seconds ( \pm standard deviation; $P<0.01$; Figure 4 ). Although the complete straining procedure for the Löffler's alkaline methylene blue method was $33.6 \pm 7.26$ seconds, that for the Diff-Quik method was $64.7 \pm 12.50$ seconds ( \pm standard deviation; $P<0.01 ;$ Figure 5).

\section{Discussion}

Smear histopathology and bacterial culture tests are being used more commonly by ophthalmologists for the diagnosis

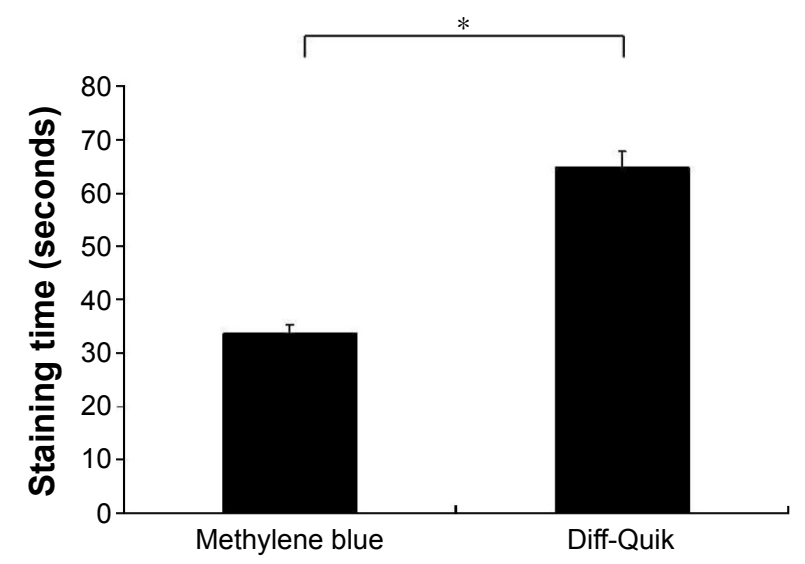

Figure 5 Comparison of whole procedure time of Löffler's alkaline methylene blue method and Diff-Quik method.

Notes: Staining time of the methylene blue method is $33.6 \pm 7.26$ seconds, while the whole procedure time of the Diff-Quik method is $64.7 \pm 12.50$ seconds ( \pm standard deviation; $* P<0.0$ I paired $t$-test). of corneal and conjunctival infections. ${ }^{6,7}$ However, the conventional staining methods require many processing steps such as the collection of smear specimens, drying, fixing, staining, and clearing. This is also true for the Diff-Quik stain which is a simplified form of Giemsa staining. In contrast, the Löffler's method with alkaline methylene blue can be done in about 30 seconds including collecting, smearing, staining with the methylene blue solution, being cover slipped, and examined. It is relatively simple and can be done on the same day as the examination. The entire procedure can be done by an ophthalmologist or an ophthalmic technician.

Even though this is a one color staining, it is possible to determine whether the infection is of bacterial or viral origin by examining whether there is a predominance of neutrophils or lymphocytes. An advantage of this rapid diagnosis method is that it is possible to prescribe an appropriate antibiotic, and it is possible to determine whether it is effective. If it is not effective, then the antibiotic can be switched. In addition, culturing and isolation can be done at the same time for more accurate identification of the microorganism.

Generally, it is difficult for stains to penetrate live cells because the cell membranes form a barrier. Therefore, cells need to be fixed before the staining. On the other hand, methylene blue does not require fixation of the cells for staining, because it penetrates into intracellular structure across cell membranes directly as a supravital stain.

Gram staining is useful for identifying the offending bacteria because it is possible to differentiate Gram-positive from Gram-negative cocci-bacilli. ${ }^{7}$ In contrast, we cannot get any further information other than a form of bacteria by the Löffler's alkaline methylene blue method which is a limitation. Furthermore, we cannot preserve the specimens by this method because nuclear staining becomes stronger over time because it was not dried or fixed. However, digital imaging will allow a long-term preservation of the images (Figure 6), ${ }^{8}$ and they can be used for informed consent by showing them to a patient. ${ }^{9}$

In conclusion, the Löffler's methylene blue method is a rapid method that can be used in the everyday clinic. It is possible to observe whether the offending bacteria are being phagocytosed by neutrophils. In contrast, the main inflammatory cells in viral conjunctivitis are lymphocytes, although it cannot be determined whether this is an epidemic type of conjunctivitis. Although more studies are necessary, it is possible to distinguish whether it is bacterial or viral conjunctivitis by staining the eye discharge with Löffler's alkaline methylene blue. 

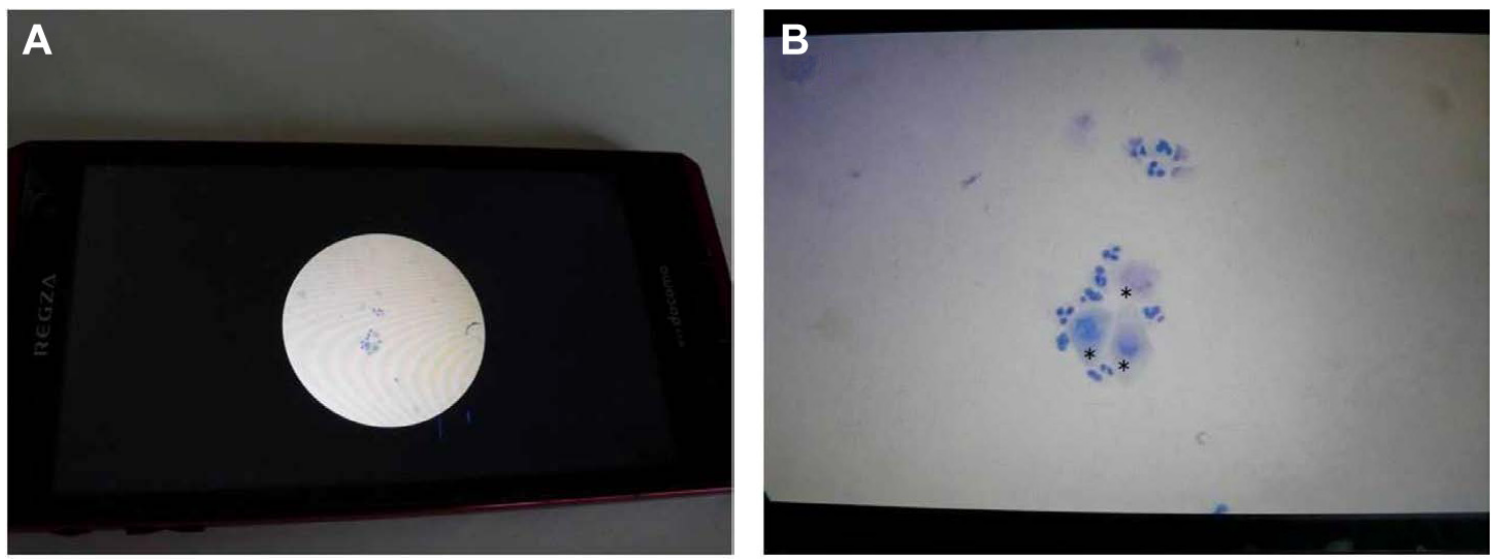

Figure 6 Images of neutrophils and conjunctival epithelium cells $\left(^{*}\right)$ in eye discharge in a patient with acute conjunctivitis taken with a smartphone.

Notes: The eye discharge is stained blue by Löffler's alkaline methylene blue method. $(\mathbf{A})$ is original size. The conventional optical microscope magnification is $\times 400$, and (B) shows magnification of $(\mathbf{A})$ by several fold.

\section{Disclosure}

The author reports no conflicts of interest in this work.

\section{References}

1. Yanoff M, Fine BS. Basic Principles of Pathology. Ocular Pathology. 5th ed. St Louis: Mosby; 2002:1-28.

2. Yanoff M, Fine BS. Conjunctiva. Ocular Pathology. 5th ed. St Louis: Mosby; 2002:215-240.

3. Weiss A, Brinser JH, Nazar-Stewart V. Acute conjunctivitis in childhood J Pediatr. 1993;122(1):10-14.

4. Spencer WH. Ophthalmic Pathology, an Atlas and Textbook. 3rd ed Philadelphia: W.B. Saunders Co.; 1985:128-150.
5. Nishizawa $\mathrm{K}$, Hatano $\mathrm{H}$. [What kind of bacteria cause conjunctivitis in Japanese patients]. Atarashii Ganka (J Eye). 2009;26(special edition): 65-68. Japanese.

6. Yamada N, Hatano H. [The point of Smear for corneal infection] Atarashii Ganka (J Eye). 2009;26(special edition):14-16. Japanese.

7. Nakagawa H, Hatano H. [Atlas of smear for ophthalmologist]. 1st ed. Tokyo: Infront Co.; 2010:15. Japanese.

8. Roy S, Pantanowitz L, Amin M, et al. Smartphone adapters for digital photomicrography. J Pathol Inform. 2014;5(1):24.

9. Kimura M, Enoki E, Maenishi O, Ito A, Chikugo T. [Capture of histopathological images by medical students using the digital cameras of cell phones and smart phones during histopathology classes]. Igakukyouiku. 2013;44(2):85-87. Japanese.
Clinical Ophthalmology

\section{Publish your work in this journal}

Clinical Ophthalmology is an international, peer-reviewed journal covering all subspecialties within ophthalmology. Key topics include: Optometry; Visual science; Pharmacology and drug therapy in eye diseases; Basic Sciences; Primary and Secondary eye care; Patient Safety and Quality of Care Improvements. This journal is indexed on

\section{Dovepress}

PubMed Central and CAS, and is the official journal of The Society of Clinical Ophthalmology (SCO). The manuscript management system is completely online and includes a very quick and fair peer-review system, which is all easy to use. Visit http://www.dovepress.com/ testimonials.php to read real quotes from published authors. 